\title{
A Quasi-Concertina Force-Displacement MEMS probe for measuring
}

\section{Biomechanical properties}

David Grech $_{1,2}{ }^{*}$, Antulio Tarazona ${ }_{1}$, Maria Theresa De Leon ${ }_{1}$, Kian S. Kiang, Jurgita Zekonyte $_{2,3}$, Robert J.K. Wood 2 , Harold M.H. Chong 1

1. School of Electronics and Computer Science, University of Southampton, Southampton, SO17 1BJ, UK

2. National Centre for Advanced Tribology, Faculty of Engineering and the Environment, University of Southampton, Southampton, SO17 1BJ, UK

3. School of Engineering, University of Portsmouth, Portsmouth, PO1 3DJ, UK

*Email: $\underline{\text { dg50ecs.soton.ac.uk }}$

Keywords: Microelectromechanical systems (MEMS); Piezoresistors, Quasi-Concertina (QC); Atomic Force Microscopy (AFM); Force - Displacement (F-D) Sensors; Biomechanics of cells

\begin{abstract}
In this work, we present the development of a novel Quasi-Concertina (QC) microelectromechanical systems (MEMS) force - displacement (F-D) sensor with a resolution as small as $5.6 \mathrm{nN}$, and $1.25 \mathrm{~nm}$, and a range of as much as $5.5 \times 10^{-3} \mathrm{~N}$, and $1000 \mu \mathrm{m}$. The performance of the microfabricated proof-of-concept QC MEMS devices are in good agreement with our analytical and numerical estimates. F-D sensors with these attributes will enable the mechanical properties of biological phenomena to be continuously measured over large F-D ranges without the need to alter the measurement instrument.
\end{abstract}

\section{Introduction}

The development of diagnostic tools, prophylactics and therapeutics of diseases frequently coincides with an increased understanding of the biomechanics of cells and tissue [1-7]. Current research activities are focusing on improving our understanding of cell biology by investigating how mechanical inputs are converted into biological and biochemical responses (mechanotransduction). These biological responses include cell motility, apoptosis, morphogenesis, adhesion, and differentiation [8-10]. Other work is looking at understanding the biological or biochemical process, external environment, or pathogens that cause the biological cells to mutate or malfunction and instigate diseases [10-13] such as glaucoma, 1 
malaria, sickle cell anaemia, arthritis, elliptocytosis, cancer, asthma, cardiovascular diseases, and spherocytosis $[4-6,9,12,14,15]$. Another area of ongoing interest is the structural changes within the cytoskeleton and the extracellular matrix (ECM) brought about by diseases. For instance, in cancer the mechanical properties of the afflicted cells change such that they can stretch and contract enabling them to migrate and bring about tumour metastasis $[6,10]$.

The typical tools currently employed to measure biomechanical properties include micropipette aspiration (MA), atomic force microscopy (AFM), and optical traps (OT) [14, 16-21]. Of these, the AFM has a F-D resolution of $1 \mathrm{pN}$, and $1 \mu \mathrm{N}$ respectively, and the largest F-D range of as much as $0.1 \mathrm{~nm}$, and $10 \mu \mathrm{m}$ respectively. However, access to this full measurement range requires several optical cantilever changes [16, 17, 19, 22]. Furthermore, cells can have deformations of the same order of magnitude as an undeformed cell, which can be as large as $100 \mu \mathrm{m}$, and forces within an artery $10 \mathrm{~mm}$ long, and with a diameter of $10 \mathrm{~mm}$ are of the order of $0.5 \mu \mathrm{N}$ to $5 \mu \mathrm{N}[14,19,23]$. Thus, it is challenging to continuously measure the full range of biomechanical properties of cells, or multicellular systems such as tissue, organs, and small organisms using the present state of the art techniques.

In previous attempts to study the mechanics of single cells at large deformations, Saif et al. used an optical microscope to measure the displacements of a customised silicon microfabricated cantilever $[3,24]$. This technique was developed further by using a twoparallel fixed-fixed beam probe, and later by using a serpentine spring [25-28]. The latter had a force resolution and F-D range of $50 \mathrm{pN}, 1 \mu \mathrm{m}$, and $3 \mathrm{~mm}$ respectively and lead to an improved understanding of cell mechanics $[22,29,30]$. In an alternative approach, Park et al. circumvented the use of a microscope to measure displacement by using self-sensing cantilevers. This achieved a force resolution of $12 \mathrm{nN}$, and a F-D range of $10^{-3} \mathrm{~N}$, and $100 \mu \mathrm{m}$ respectively and was successfully used to investigate mechanotransduction mechanisms [17, $31,32]$.

In this work we propose adding Hot Wire Chemical Vapour Deposition (HWCVD) piezoresistors to Quasi Concertina (QC) springs, both developed by the authors in earlier work [33, 34], to address the challenges of continuously measuring the biomechanical properties of cells and tissue with a $\mathrm{nN}$ and $\mathrm{nm}$ resolution, with a F-D range greater than $10^{-3}$ $\mathrm{N}$ and $100 \mu \mathrm{m}$. The large displacement, and high linearity, in conjunction with a novel 
method used to integrate self-sensing, make the QC spring ideal for this application. The envisaged use of the device entails functionalising the suspended structure with the addition of a probe. The probe could either be included as part of the microfabrication process, or bonded on to the suspended structure using a suitable adhesive. Quasi-static indentation force or tether pulling measurements could then be achieved using techniques similar to those used with AFM. As proof of concept we have microfabricated QC MEMS sensors and shown that they can achieve a F-D resolution and range of $5.4 \mathrm{nN}-5.5 \times 10^{-3} \mathrm{~N}$, and $1.25 \mathrm{~nm}-1000 \mu \mathrm{m}$ respectively, and that the results are in good agreement with the analytical and numerical estimates.

\section{Methods}

\section{Design and optimisation of a piezo-resistive self-sensing QC MEMS}

The design of the QC spring is based on three approaches. The first is using a number of beams in series to increase the displacement range and linearity. This is demonstrated in the design of the lateral suspension accelerometer developed by Pike et al., and the MEMS sensor developed by Rajagopalan et al. $[22,29,30,35,36]$. The second approach is that the bending compliance of a beam with a thickness significantly less than its width is greatest when the applied force is perpendicular to its width. The third approach is to make the compliance of a beam in tension or compression significantly less than that of a beam in bending. Using these hypotheses, a platform was suspended with beams oriented such that a high compliance, linearity, and displacement range is achieved in the $\mathrm{z}$ out-of-plane direction as shown in the ANSYS Finite Element Analysis (FEA) model (Figure 1). In addition, the displacement of the suspended platform is restricted in the $x / y$ in-plane direction by the interconnected beams on each of the four sides of the platform. This ensures that the out-ofplane compliance is greater than the in-plane compliance [34]. 


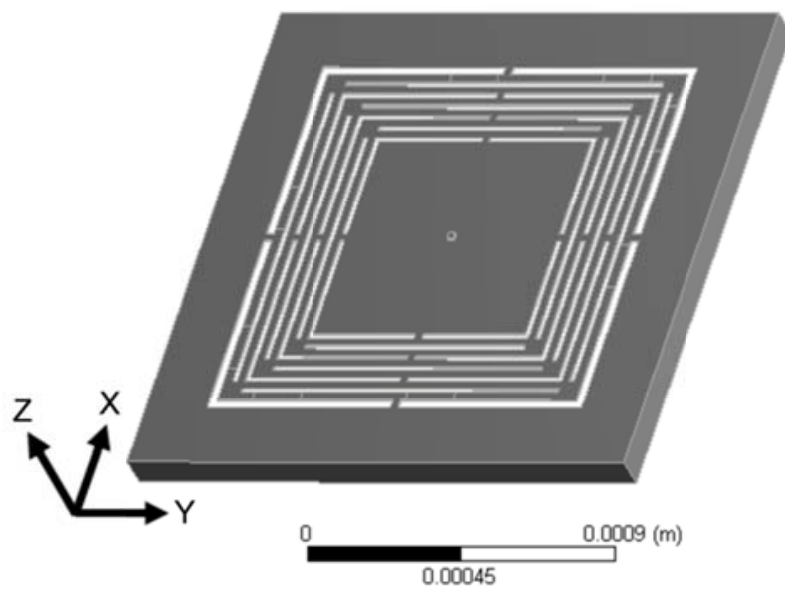

(a)

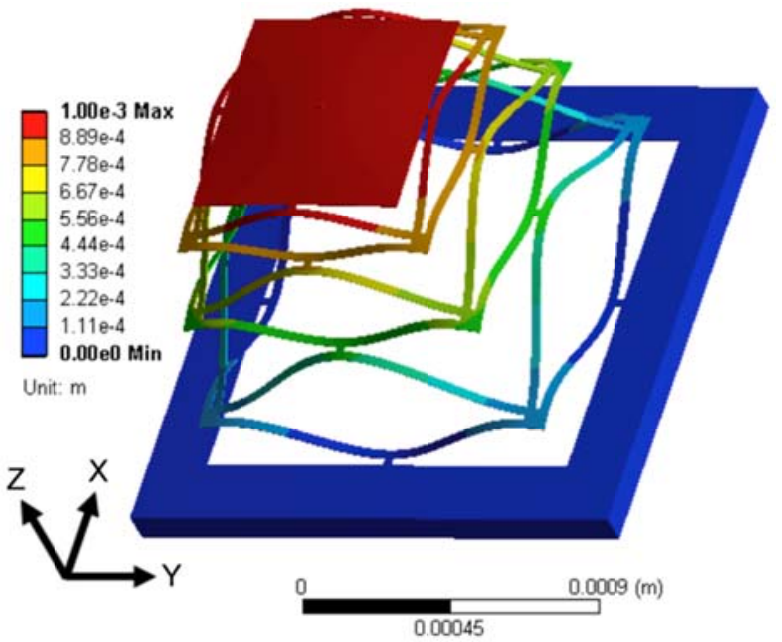

(b)

Figure 1: A (FEA) rendered model of the QC flexure showing the multiple interconnected beams, with (a) no force applied, and (b) a force (in the $-\mathrm{z}$ direction) applied to the suspended platform.

Self-sensing was integrated into the QC spring using piezoresistive transduction. The piezoresistors were positioned in a full Wheatstone bridge configuration as shown in Figure 2 to maximise the sensitivity and minimise the effect of temperature.

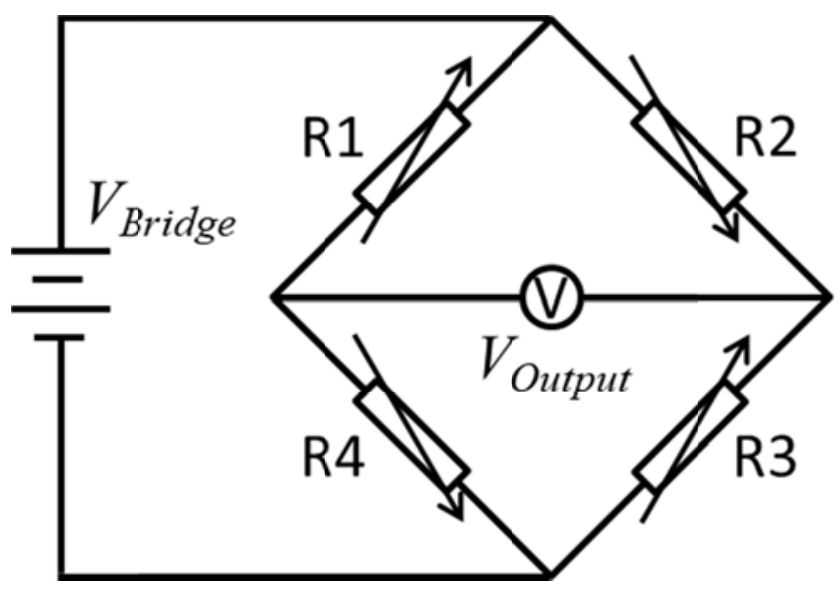

Figure 2: To achieve self-sensing, active piezoresistors R1, R2, R3, and R4 were configured into a full Wheatstone bridge and integrated onto the suspended beams of the QC spring.

The $\mathrm{V}_{\text {Bridge }}$ and $\mathrm{V}_{\text {Output }}$ shown in Figure 2 are the DC supply voltage and the measured change in voltage (the output signal) respectively. For a full Wheatstone bridge these values have the following relationship [37]:

$$
\frac{V_{\text {Output }}}{V_{\text {Bridge }}}=\frac{R_{1} R_{3}-R_{2} R_{4}}{\left(R_{1}+R_{2}\right)\left(R_{3}+R_{4}\right)}
$$

The equation indicates that the maximum $V_{\text {Output }}$ occurs when the resistance in $R_{1}$ and $R_{3}$ increases $(+\Delta R)$ and the resistance in $R_{2}$ and $R_{4}$ decreases $(-\Delta R)$ as indicated by the arrows 4 
in Figure 2. The reverse, i.e. maximum $V_{\text {Output }}$ when $R 1$ and $R 3$ decrease $(-\Delta R)$ and the resistance in $\mathrm{R} 2$ and $\mathrm{R} 4$ increases $(+\mathrm{R})$, is also true, thus the sensor can measure both positive and negative displacements. In full bridge configurations typically used on cantilevers [38] or pressure sensors [37], the equal but opposite polarity of the longitudinal and transverse piezoresistive coefficients in p-type crystalline silicon (c-Si) is used to achieve the opposing change in resistance. However, the resulting sensitivity is highly dependent on the accuracy of the position of the transverse piezoresistor relative to the stressed beam. This makes the photolithographic alignment critical to the performance of the device [37]. Moreover, unlike c-Si piezoresistors, the transverse piezoresistive coefficient in polycrystalline silicon (poly$\mathrm{Si})$ is substantially less $(<50 \%)$ than that in the longitudinal direction. Hence, using poly-Si piezoresistors in a transverse arrangement would result in a further loss in sensitivity.

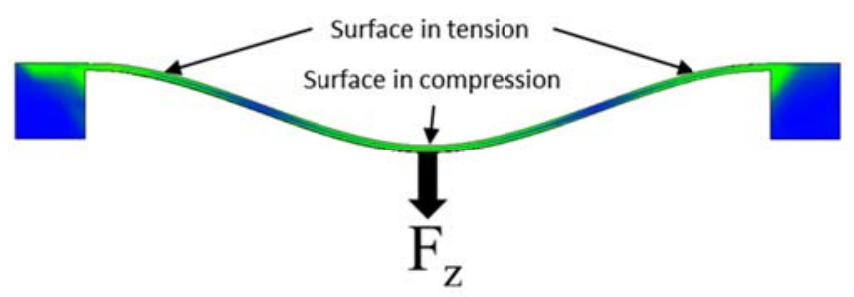

(a)

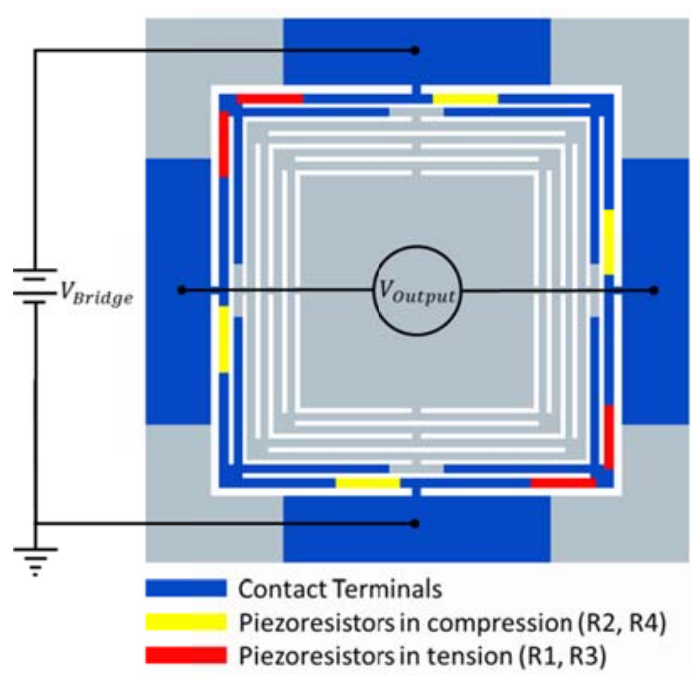

(b)

Figure 3: (a) Areas in compression and in tension of a double clamped beam with a central load, and (b) top view of a QC sensor with piezoresistors for self-sensing. The beams are in compression and in tension in the areas

indicated only when a load is applied directly into the page (z-direction). The piezoresistors are strategically positioned in areas that are in compression and in tension to fulfil the requirements for a full Wheatstone bridge. The contact terminals continue onto the second set of beams to help dissipate piezoresistor heat.

In this work, an alternative approach was taken by strategically positioning all the piezoresistors longitudinally on the beams in areas that are in compression or in tension as shown in Figure 3a. By using this arrangement, the piezoresistors in tension have an equal and opposite change in resistance to the piezoresistors in compression. To ensure the piezoresistors are matched, each of the four piezoresistors have been divided into two equal segments in series and positioned over two beams orthogonal to one another as shown in Figure $3 \mathrm{~b}$. Thus, in the event of photolithography misalignment (lateral or rotational), all the 
piezoresistors will be affected equally and the resistance bridge remains balanced. Using this approach, the resistances $\mathrm{R}_{1}$ and $\mathrm{R}_{3}$ can be written as [37]:

$$
R_{1}=R_{3}=\left(1+\pi_{l} \sigma_{l}\right) R_{0}
$$

where $\mathrm{R}_{0}, \pi_{1}$, and $\sigma_{1}$ are the resistance of the unstressed piezoresistors, longitudinal piezoresistive coefficient, and the longitudinal stress in the beam respectively. For the piezoresistors $\mathrm{R}_{2}$ and $\mathrm{R}_{4}$ under compression, the equation becomes [37]:

$$
R_{2}=R_{4}=\left(1+\pi_{l}\left(-\sigma_{l}\right)\right) R_{0}
$$

and substituting into Eq. 1 gives the ratio of the measured output voltage to the bridge voltage:

$$
\frac{V_{\text {Output }}}{V_{\text {Bridge }}}=\pi_{l} \sigma_{l}
$$

Therefore, the overall force sensitivity, $\mathrm{S}_{\mathrm{f}}$, for a $\mathrm{QC}$ sensor can be calculated using:

$$
S_{f}=\frac{V_{\text {Output }}}{F}=\frac{\pi_{l} \sigma_{l} V_{\text {Bridge }}}{F}
$$

where $\mathrm{F}$ is the force applied to the suspended structure.

\section{Optimisation}

The performance of the device was optimised using the guidelines for piezoresistive devices published by Park et al., and Doll et al. [39-42]. A total of 6 devices were optimised. QC 1, 2, and 3 were optimised for poly-Si piezoresistors, while QC 4, 5, and 6 were optimised for c-Si piezoresistors. The characteristics of these devises are shown in Table 1. The HWCVD poly-Si piezoresistors used in these estimates have a gauge factor of 44 [42]. This is significantly less than the gauge factor of a c-Si piezoresistor which could be as much as 130. Furthermore, poly-Si piezoresistors have a Hooge factor two orders of magnitude larger than that of c-Si piezoresistors [40]. This explains the significantly higher F-D resolution of the devices with c-Si piezoresistors. Additional details are provided in the Supplementary Table S1. 
Table 1: Design characteristics of the optimised QC MEMS probes

\begin{tabular}{|c|c|c|c|c|c|}
\hline \multirow{2}{*}{$\begin{array}{c}\text { Device } \\
\text { No. }\end{array}$} & Piezoresistor & \multicolumn{2}{|c|}{ Resolution } & \multicolumn{2}{c|}{ Range } \\
\cline { 3 - 6 } & Type & Force (nN) & Displ. $(\mathrm{nm})$ & Force $\left(10^{-3} \mathrm{~N}\right)$ & Displ. $(\mu \mathrm{m})$ \\
\hline QC1 & poly-Si & 6.3 & 0.93 & 6.3 & 1000 \\
\hline QC2 & poly-Si & 0.78 & 4.31 & 1.3 & 7250 \\
\hline QC3 & poly-Si & 0.86 & 4.77 & 1.4 & 8000 \\
\hline QC4 & c-Si & 0.052 & 0.019 & 6.3 & 1000 \\
\hline QC5 & c-Si & 0.020 & 0.111 & 1.3 & 7250 \\
\hline QC6 & c-Si & 0.019 & 0.106 & 1.4 & 8000 \\
\hline
\end{tabular}

\section{Fabrication}

Proof-of-concept sensors, based on the less intricate QC 1 design, were microfabricated and characterised to validate the design. The fabrication process is included in the Supplementary Information. Details of the HWCVD poly-Si piezoresistors used in this work are available in previously published work by the author [33]. Figure 4 shows scanning electron microscope (SEM) images of a microfabricated QC flexure with the integrated full Wheatstone bridge.

Analytical and numerical methods used to estimate the spring constant and displacement limit

The analytical method to estimate the spring constant of the QC spring was described previously [34]. Briefly, the spring is deconstructed into individual double clamped beams, and the spring constant for each resultant individual beam estimated using Bernoulli-Euler beam theory. The total spring constant was than estimated by accounting for each beam in the system according to whether they are in parallel or in series. The numerical methods used to estimate the spring constant, linearity, and displacement limit are available in the Supplementary Information.

\section{Methods used to measure the spring constant}

The spring constant of the QC springs were measured as described previously [34]. Briefly, the spring constant of the QC spring for displacements $\leq 5 \mu \mathrm{m}$ was measured using a modified atomic force microscope cantilever-on-cantilever method [43]. A F-D rig, consisting of a precision balance positioned directly beneath a Vernier micrometer head, was 
employed for measurements at displacements $>5 \mu \mathrm{m}$. Additional detail about these methods is available in the Supplementary Information.

\section{Methods used to measure the electrical characteristics}

For the electrical characterisation, the devices were mounted onto bespoke connectors using Crystalbond and electrical connections were made using aluminium wire bonding as shown in Figure 4d and Supplementary Figure S16. The devices were then connected to a circuit which powered the bridge and amplified the output signal. The circuit (Supplementary Figure S15) consisted of a precision amplifier, with buffered coarse and fine reference voltage adjustment and a voltage regulator with buffered bridge voltage adjustment. The circuit allowed measurements to be performed using the $+15 /-15 \mathrm{~V}$ source available from the AFM or using batteries as a low noise power supply. For sensitivity measurements with displacements $\leq 5 \mu \mathrm{m}$, the devices were connected to an AFM (Bruker, Multimode V) using a bespoke adapter in place of the microscope's laser head (Supplementary Figures S18-20). To actuate the devices, a $100 \mu \mathrm{m}$ diameter by $2 \mathrm{~mm}$ length of piano wire was bonded perpendicularly to a metal sample disc $(10 \mathrm{~mm}$ diameter $\mathrm{x} 0.5 \mathrm{~mm}$ thick $)$ using a cyanoacrylate adhesive and mounted on to the microscope's sample stage (Supplementary Figure S21). The output from the device was then recorded using the AFM. For displacements $>5 \mu \mathrm{m}$ the forces applied to the device were measured using the F-D rig. In this setup, the device was actuated using a Vernier micrometer head connected to the device's spring platform via a $100 \mu \mathrm{m}$ diameter by $10 \mathrm{~mm}$ length of piano wire bonded at both ends using a cyanoacrylate adhesive. During the Power Spectral Density (PSD) noise characterisation measurements, the device and the circuit were placed inside a shielded aluminium enclosure to reduce the influence of environmental noise. The bridge voltage was provided through a battery source and the amplified output from the device was connected to a RF spectrum analyser [33]. Further details are provided in the Supplementary Information. 


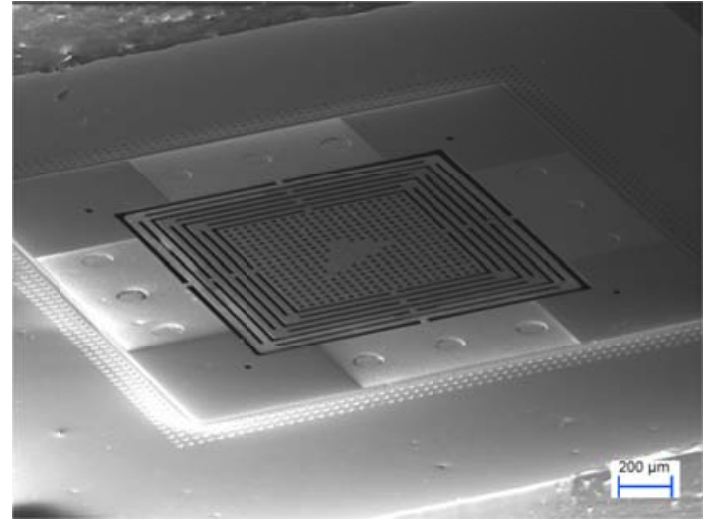

(a)

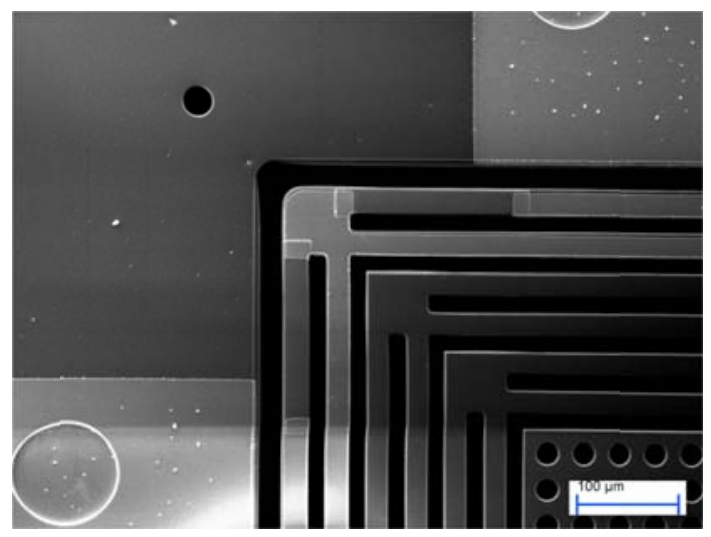

(c)

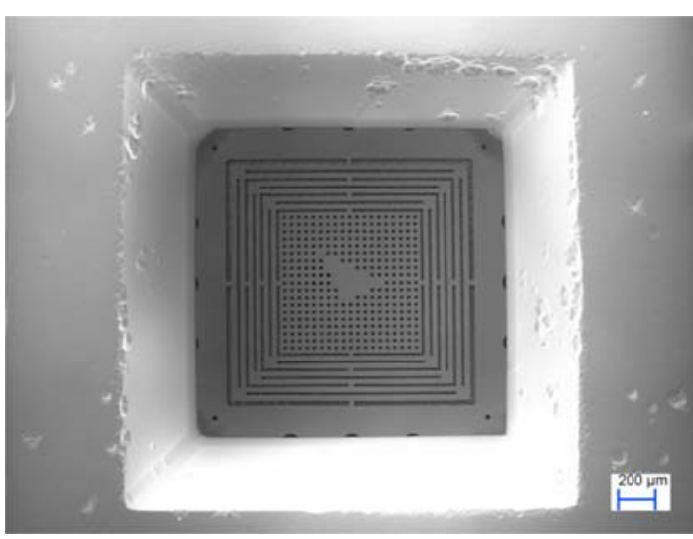

(b)

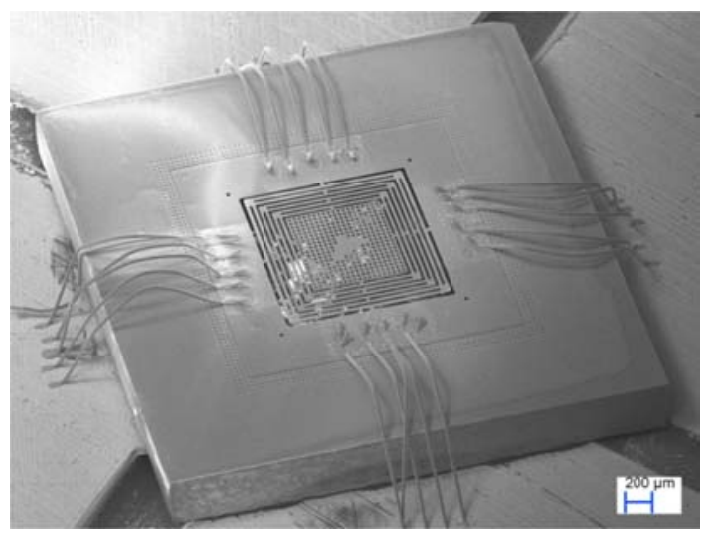

(d)

Figure 4: SEM images of (a) an isometric view, (b) bottom view and (c) piezoresistors of a self-sensing QC flexure (QC1). Surface particles are from the Crystalbond. These do not affect the performance of the device. (d) SEM image of a QC sensor (QC1) mounted onto an electrical connector using Crystalbond and wire bonding. The holes on the suspended platform were incorporated into the design for a microfabrication process (hydrofluoric $\mathrm{SiO}_{2}$ etch release of the platform from the buried oxide layer). This technique was not ideal for this application and was abandoned; however, we continued to use the lithographic patterns. The asymmetric triangular pattern made by the holes on the suspended platform was made in the eventuality the fabrication of sharp silicon tips, as described by Burt et al. [44], was required.

\section{Results and discussion}

The measured spring constant for the QC 1 flexure was $4.69 \mathrm{~N} / \mathrm{m}, 5.39 \mathrm{~N} / \mathrm{m}$, and 7.34 $\mathrm{N} / \mathrm{m}$ at displacements of $5 \mu \mathrm{m}, 100 \mu \mathrm{m}$, and $1 \mathrm{~mm}$ respectively. The error between the spring constant estimated using a numerical method, developed specifically for the application using MATLAB, and the measurements are within $23 \%, 12 \%$, and $4 \%$ for the same displacements respectively (refer to Supplementary Figure S14). The large error in the spring constant at a displacement of $5 \mu \mathrm{m}$ can be in part attributed to the inherent error in the cantilever-oncantilever method which can be as much as $30 \%$ [43], and due to the microfabricated device not having the exact design intended beam thickness and width. Multiple F-D measurements 
(Figure 5.a) were carried out to investigate the hysteresis of the device. The displacements were limited to $\leq 700 \mu \mathrm{m}$ to avoid damaging the device. The measurements indicate that the hysteresis approaches $0 \%$ for displacements $\leq 700 \mu \mathrm{m}$. This is typical for devices fabricated from a brittle material such as Si. The measured mechanical non-linearity was $1 \%$ for displacements $\leq 100 \mu \mathrm{m}$, and 10\% for displacements $>100 \mu \mathrm{m}$ and $\leq 700 \mu \mathrm{m}$ (Figure 5.b).

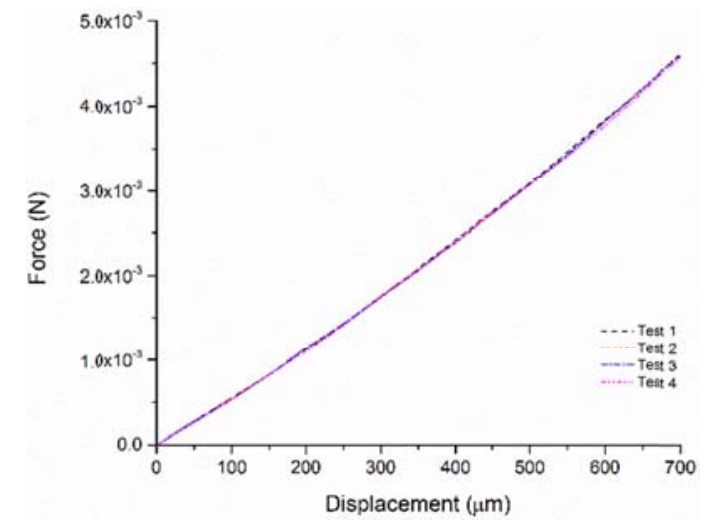

(a)

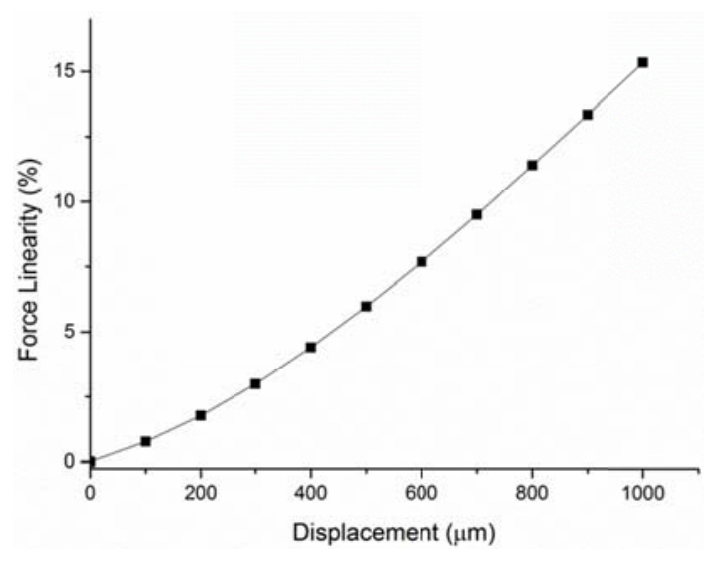

(b)

Figure 5: Representative QC1 spring measurements (a) F-D hysteresis (multiple load cycles), and (b) Force linearity versus displacement (loaded until fracture) graphs

The total measured displacement before fracture for QC 1 was as much as $1000 \mu \mathrm{m}$ which is within $5 \%$ of the estimates made using finite element analysis (FEA) software (ANSYS). Figure 6 shows a scanning electron microscope (SEM) image of a QC spring being displaced using a needle tip. This shows the concertina-like beam deflections which enable the device to have such large deflections. The geometry of the beam deflections is similar to that produced by FEA (results shown as a rendered image in Figure 1b). The tilt shown is due to the tip of the needle not being applied exactly to the centre of the suspended platform. In the application context the sensor will have a probe mounted centrally and normal to the suspended platform and displacement will be controlled using piezoelectric stacks such as those employed on AFM stages. The probe will then be brought into contact perpendicularly to the surface of the specimen. This arrangement, which is not unlike that used in an AFM, will minimise sensor tilt.

A modal analysis of the structure using FEA software (ANSYS), and characterisation of the device, show that the fundamental mode of the device was $>4.5 \mathrm{KHz}$ [34], thus outside the quasi-static measurement frequency $(\leq 10 \mathrm{~Hz})$ envisioned for this device. 


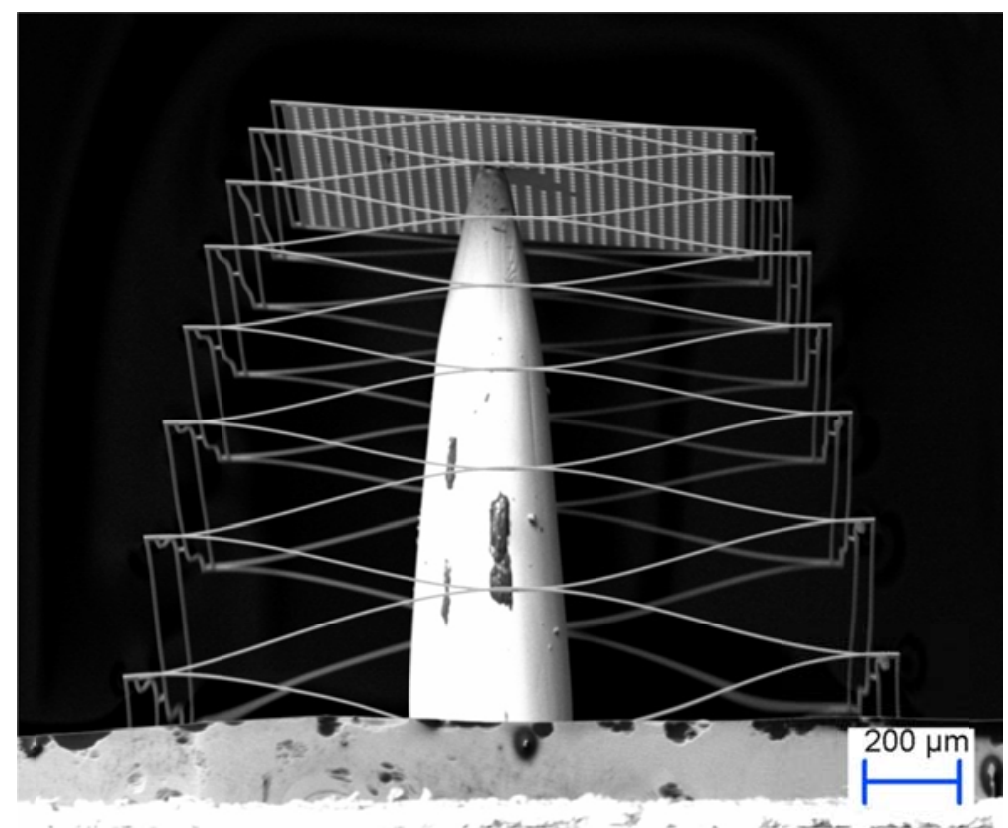

Figure 6: SEM image showing the large deflection capabilities of a QC spring (QC5) which has a beam thickness of $4.5 \mu \mathrm{m}$, and a beam width of $10 \mu \mathrm{m}$. To demonstrate the range of the QC MEMS, the device was mounted on a soft vinyl block using cyanoacrylate adhesive. A needle was inserted into the vinyl block and used to deflect the suspended platform as shown. Since Si does not exhibit any plastic deformation [4.5], the spring returns to its un-deflected state following the removal of the applied force.

The resolution in piezoresistive transduction is limited by the noise generated by the piezoresistors, and the electronics system used to amplify the signal. A representative noise power spectral density (PSD) plot for a fabricated QC 1 sensor is shown Figure 7a. The noise is greater at lower frequencies and becomes uniform at frequencies $>10 \mathrm{KHz}$. This is typical of Hooge noise which is the dominant source of noise in piezoresistors.

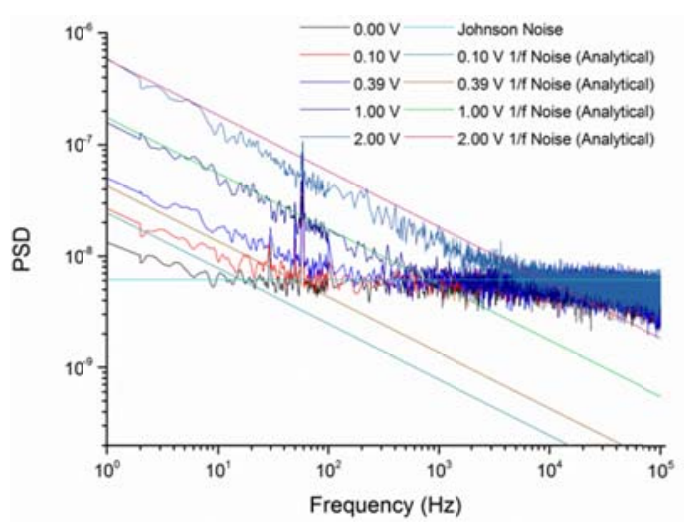

(a)

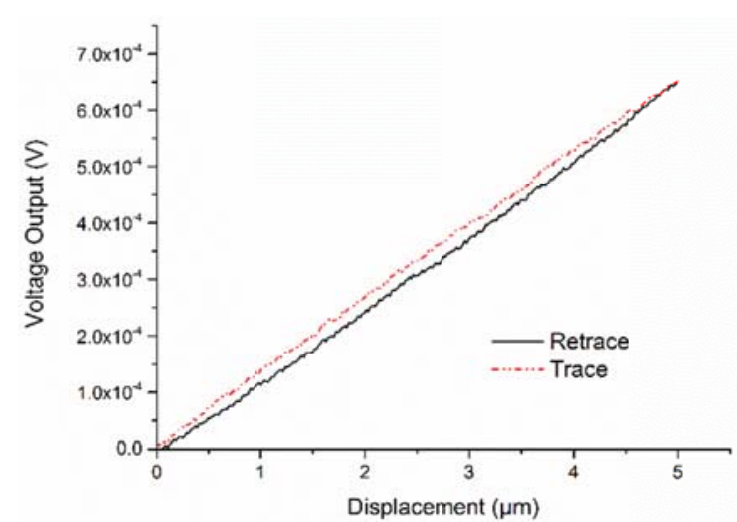

(b) 


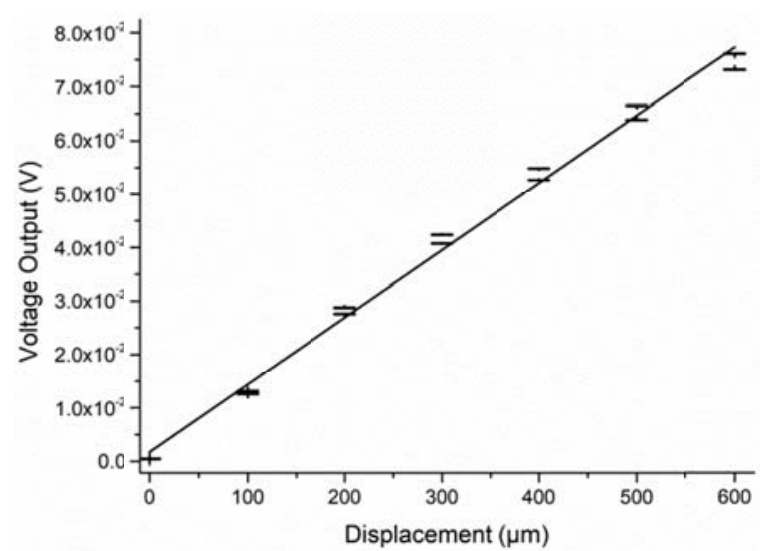

(c)

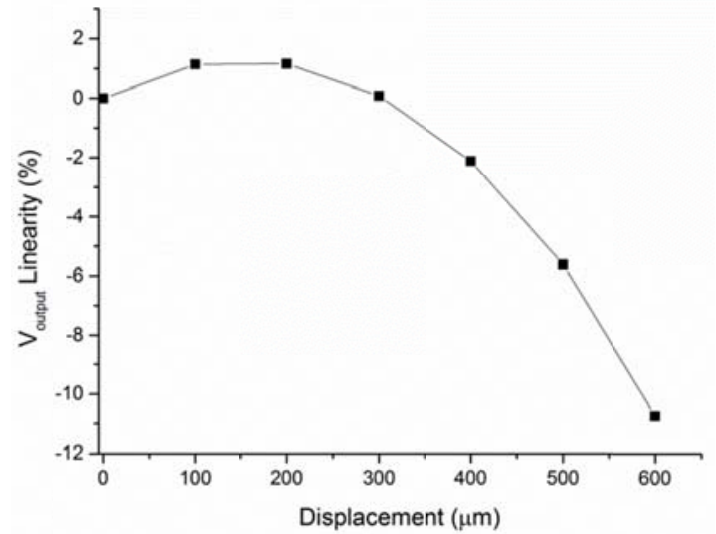

(d)

Figure 7: Representative (a) noise PSD for QC 1 at various voltages, and (b) calibration sensitivity curves for QC 1 measured using an AFM microscope in F-D measurement mode. The curve shows hysteresis which may be due compliance in the piano wire used to actuate it. Representative (c) calibration sensitivity curves for QC 1 for displacements as large as $600 \mu \mathrm{m}$, and (d) $\mathrm{V}_{\text {Output }}$ linearity \% versus the displacement of QC 1.

At higher frequencies, the Johnson noise which is independent of both the frequency and the bridge voltage, becomes the dominant source of noise [39-41]. The estimated Hooge and Johnson noise, at various bridge voltage, are in good agreement with the measurements.

The trace and retrace plots of the $V_{\text {Output }}$ versus displacement graph (Figure $7 b$ ) measured using the AFM setup indicate a hysteresis of $3.82 \%$ at a displacement of $2.5 \mu \mathrm{m}$ which is higher than expected. The likely cause for this hysteresis is compliance in the piano wire used to actuate the device. The gradient of the curve represents the relationship between displacement of the suspended platform and the measured voltage output and is known as the displacement sensitivity of the device.

A further $V_{\text {Output }}$ versus displacement plot, this time measured using the F-D rig, is shown in Figure 7c. These measurements were also repeated 5 times to investigate the hysteresis of the device. The measured $\mathrm{V}_{\text {Output }}$ versus displacement hysteresis is $0.73 \%$ which is significantly less than that measured using the AFM setup. This supports the hypotheses that the hysteresis observed in the AFM measurements originates from the test method and not the device. The measured $\mathrm{V}_{\text {Output }}$ versus the displacement non-linearity is $1 \%$ and $10 \%$ for displacements of $100 \mu \mathrm{m}$ and $575 \mu \mathrm{m}$ respectively (Figure $7 \mathrm{~d}$ ). An overall linearity, achieved by superimposing the measured force and electrical output linearity, estimates the device linearity to be $\leq 1 \%$, and $<+/-3 \%$ over a displacement of $75 \mu \mathrm{m}$, and $600 \mu \mathrm{m}$ respectively (Figure 8). 


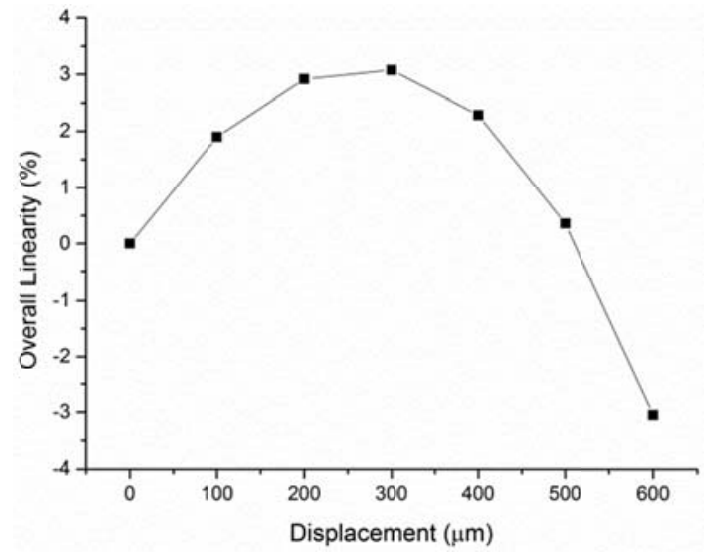

Figure 8: Combined mechanical and electrical linearity versus displacement of the device. This was estimated by superimposing the measured force and electrical output linearity.

The displacement and force sensitivity versus the bridge voltage for QC 1 is shown in Figure 9a. The plot was prepared using data from the AFM setup (displacements $\leq 5 \mu \mathrm{m}$ ) and the F-D rig setup (displacements $\leq 600 \mu \mathrm{m}$ ) F-D calibration sensitivity curves.

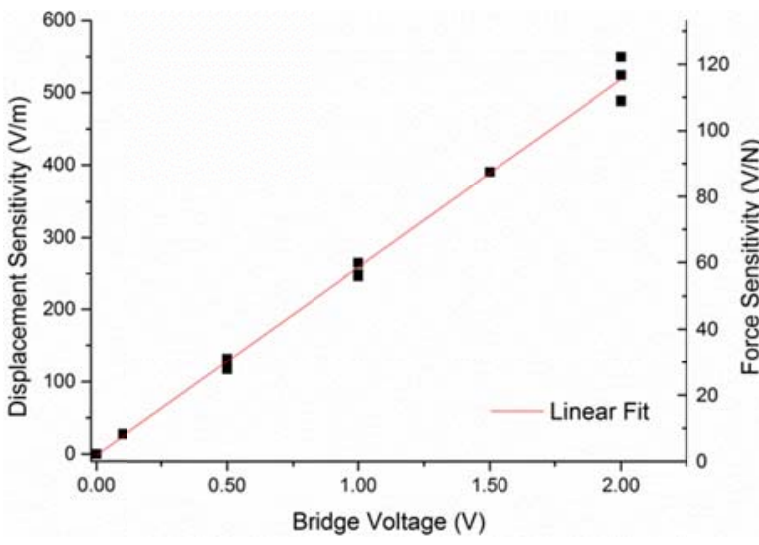

(a)

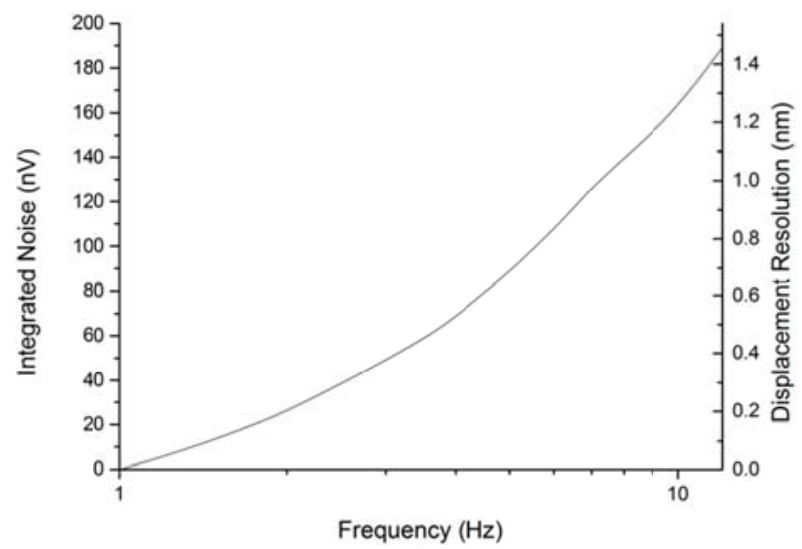

(b)

Figure 9: (a) Displacement and force sensitivity versus the bridge voltage for QC 1 . The data for the graphs was extracted from the micro and macro sensitivity measurements, and (b) representative integrated noise and displacement resolution versus the frequency for measurements made with a bridge voltage of $0.39 \mathrm{~V}$ for QC 1

The linear change in sensitivity versus the change in the bridge voltage is as expected for a piezoresistive device [42]. At a bridge voltage of $0.39 \mathrm{~V}, \mathrm{QC} 1$ has a force sensitivity of $22.4 \mathrm{~V} / \mathrm{N}$ which is within $2 \%$ of the estimates. The force resolution is the minimum force that can be detected just above the electrical noise. This can be estimated by dividing the integrated noise at the frequency of interest by the force sensitivity (shown graphically in Figure 9a). For measurements made with a bridge voltage of $0.39 \mathrm{~V}$, the force and displacement resolution at $1-10 \mathrm{~Hz}$ is $5.6 \mathrm{nN}$, and $1.25 \mathrm{~nm}$ respectively which are within $5 \%$ 
and $29 \%$ of our estimates. The error in the displacement resolution was mainly due to the difference between the spring constant estimate and the measured spring constant.

\section{Conclusions}

In this work we have shown that a highly linear large displacement MEMS sensor with predicable mechanical and electrical characteristics, suitable for continuously measuring the biomechanical properties of cells and tissue with $\mathrm{nN}$ and $\mathrm{nm}$ resolution, and with a F-D range greater than $10^{-3} \mathrm{~N}$ and $100 \mu \mathrm{m}$, can be achieved by integrating a full Wheatstone bridge on to a QC spring. Furthermore, we can predict that similar devices will be capable of a F-D resolution of at least one order of magnitude higher. Future research will focus on the fabrication of higher resolution devices, and the use of the QC MEMS sensors for measuring the mechanical properties of Biomaterials.

\section{Acknowledgement}

This project is being funded by the Faculty of Engineering and the Environment (FEE) of the University of Southampton, the European Social Fund (ESF) and the Government of Malta. The author would like to acknowledge the Electronics and Computer Science Mechanical Workshop, and Southampton Nanofabrication Centre at the University of Southampton.

\section{References}

1. Suresh, S., et al., Reprint of: Connections between single-cell biomechanics and human disease states: gastrointestinal cancer and malaria. Acta Biomaterialia, 2015. 23, Supplement: $\mathrm{p}$. S3-S15.

2. Lekka, M., Atomic Force Microscopy a Tip for Diagnosing Cancer. Nature Nanotechnology, 2012. 7(11): p. 691-692.

3. Norman, J.J., et al., Microsystems for Biomechanical Measurements. Pediatr Res, 2008. 63(5): p. 576-583.

4. Xu, W.W., et al., Cell Stiffness Is a Biomarker of the Metastatic Potential of Ovarian Cancer Cells. Plos One, 2012. 7(10).

5. Tian, M., et al., The nanomechanical signature of liver cancer tissues and its molecular origin. Nanoscale, 2015. 7(30): p. 12998-3010.

6. Plodinec, M., et al., The nanomechanical signature of breast cancer. Nature Nanotechnology, 2012. 7(11): p. 757-65.

7. Zhang, G., et al., Design of the MEMS Piezoresistive Electronic Heart Sound Sensor. Sensors (Basel, Switzerland), 2016. 16(11): p. 1728.

8. Trichet, L., et al., Evidence of a large-scale mechanosensing mechanism for cellular adaptation to substrate stiffness. Proc Natl Acad Sci U S A, 2012. 109(18): p. 6933-8.

9. Lim, C.T., et al., Experimental techniques for single cell and single molecule biomechanics. Materials Science and Engineering: C, 2006. 26(8): p. 1278-1288. 
10. Suresh, S., Biomechanics and biophysics of cancer cells. Acta Biomater, 2007. 3(4): p. 413-38.

11. Nikkhah, M., et al., Engineering microscale topographies to control the cell-substrate interface. Biomaterials, 2012. 33(21): p. 5230-46.

12. Lee, G.Y. and C.T. Lim, Biomechanics approaches to studying human diseases. Trends Biotechnol, 2007. 25(3): p. 111-8.

13. Discher, D., et al., Biomechanics: cell research and applications for the next decade. Ann Biomed Eng, 2009. 37(5): p. 847-59.

14. Addae-Mensah, K.A. and J.P. Wikswo, Measurement techniques for cellular biomechanics in vitro. Experimental Biology and Medicine, 2008. 233(7): p. 792-809.

15. Last, J.A., et al., The Applications of Atomic Force Microscopy to Vision Science. Investigative Ophthalmology \& Visual Science, 2010. 51(12): p. 6083-6094.

16. Loh, O., A. Vaziri, and H.D. Espinosa, The Potential of MEMS for Advancing Experiments and Modeling in Cell Mechanics. Experimental Mechanics, 2009. 49(1): p. 105-124.

17. Park, S.J., M.B. Goodman, and B.L. Pruitt, Analysis of nematode mechanics by piezoresistive displacement clamp. Proceedings of the National Academy of Sciences of the United States of America, 2007. 104(44): p. 17376-17381.

18. Van Vliet, K.J., G. Bao, and S. Suresh, The biomechanics toolbox: experimental approaches for living cells and biomolecules. Acta Materialia, 2003. 51(19): p. 5881-5905.

19. Kim, D.H., et al., Microengineered Platforms for Cell Mechanobiology. Annual Review of Biomedical Engineering, 2009. 11: p. 203-233.

20. Donald, J.S., et al., Nanomechanical force transducers for biomolecular and intracellular measurements: is there room to shrink and why do it? Reports on Progress in Physics, 2015. 78(2): p. 024101.

21. Meyers, M.A., et al., Biological materials: Structure and mechanical properties. Progress in Materials Science, 2008. 53(1): p. 1-206.

22. Rajagopalan, J., A. Tofangchi, and M. Saif, Linear High-Resolution BioMEMS Force Sensors With Large Measurement Range. Journal of Microelectromechanical Systems, 2010. 19(6): p. 1380-1389.

23. Bao, G. and S. Suresh, Cell and molecular mechanics of biological materials. Nature Materials, 2003. 2(11): p. 715-725.

24. Saif, M., C. Sager, and S. Coyer, Functionalized Biomicroelectromechanical Systems Sensors for Force Response Study at Local Adhesion Sites of Single Living Cells on Substrates. Annals of Biomedical Engineering, 2003. 31(8): p. 950-961.

25. Yang, S.Y. and T. Saif, Reversible and repeatable linear local cell force response under large stretches. Experimental Cell Research, 2005. 305(1): p. 42-50.

26. Yang, S. and M. Saif, Force response and actin remodeling (agglomeration) in fibroblasts due to lateral indentation. Acta Biomaterialia, 2007. 3(1): p. 77-87.

27. Yang, S.Y. and M. Saif, MEMS based force sensors for the study of indentation response of single living cells. Sensors and Actuators a-Physical, 2007. 135(1): p. 16-22.

28. Yang, S. and M. Saif, Microfabricated Force Sensors and Their Applications in the Study of Cell Mechanical Response. Experimental Mechanics, 2009. 49(1): p. 135-151.

29. Rajagopalan, J. and M. Saif, MEMS sensors and microsystems for cell mechanobiology. Journal of Micromechanics and Microengineering, 2011. 21(5).

30. Rajagopalan, J., A. Tofangchi, and M. Saif, Highly Linear, Ultra Sensitive Bio-Mems Force Sensors with Large Force Measurement Range. Mems 2010: 23rd leee International Conference on Micro Electro Mechanical Systems, Technical Digest, 2010: p. 88-91.

31. Park, S.-J., Development of piezoresistive microcantilever based force feedback system for analysis of mechanosensation in caenorhaditis elegans nematodes, in Mechanical engineering. 2009, STANFORD UNIVERSITY. p. 140 pages. 
32. Park, S.J., M.B. Goodman, and B.L. Pruitt, Measurement of mechanical properties of Caenorhabditis elegans with a piezoresistive microcantilever system. 2005 3rd IEEE/EMBS Special Topic Conference on Microtechnology in Medicine and Biology, 2005: p. 400-403.

33. Grech, D., et al., Large Gauge Factor of Hot Wire Chemical Vapour Deposition In-Situ Boron Doped Polycrystalline Silicon. Materials Research Express, 2016.

34. Grech, D., et al., Highly linear and large spring deflection characteristics of a QuasiConcertina MEMS device. Microelectronic Engineering, 2014. 119(0): p. 75-78.

35. Pike, W.T. and S. Kumar, Improved design of micromachined lateral suspensions using intermediate frames. Journal of Micromechanics and Microengineering, 2007. 17(8): p. 1680-1694.

36. Pike, W.T., I.M. Standley, and W.B. Banerdt, A high-sensitivity broad-band seismic sensor for shallow seismic sounding of the lunar regolith. 2005.

37. Senturia, S.D., Microsystem design. 2000, Boston: Kluwer Academic Publishers.

38. Mallon, J.R., et al., Low $1 /$ f noise, full bridge, microcantilever with longitudinal and transverse piezoresistors. Applied Physics Letters, 2008. 92(3).

39. Park, S.J., J.C. Doll, and B.L. Pruitt, Piezoresistive Cantilever Performance-Part I: Analytical Model for Sensitivity. Journal of Microelectromechanical Systems, 2010. 19(1): p. 137-148.

40. Park, S.J., et al., Piezoresistive Cantilever Performance-Part II: Optimization. Journal of Microelectromechanical Systems, 2010. 19(1): p. 149-161.

41. Doll, J.C., S.J. Park, and B.L. Pruitt, Design optimization of piezoresistive cantilevers for force sensing in air and water. Journal of Applied Physics, 2009. 106(6).

42. Doll, J.C. and B. Pruitt, Piezoresistor design and applications. 1 ed. Microsystems and Nanosystems. 2013: Springer-Verlag New York. 1 online resource (xi, 245 pages).

43. Kim, M.-S., et al., Accurate determination of spring constant of atomic force microscope cantilevers and comparison with other methods. Measurement, 2010. 43(4): p. 520-526.

44. Burt, D.P., et al., A simple method for high yield fabrication of sharp silicon tips. Microelectronic Engineering, 2008. 85(3): p. 625-630.

45. Madou, M.J., Fundamentals of microfabrication : the science of miniaturization. 2nd ed. 2002, Boca Raton, Fla.: CRC Press. 723 p., 16 p. of plates. 